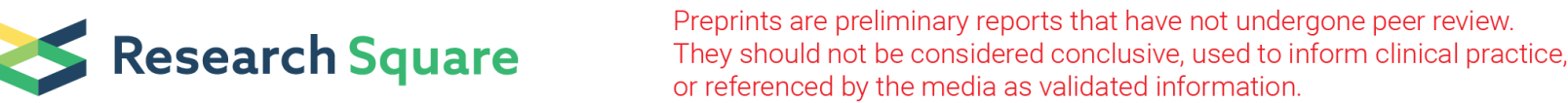

\section{Circular RNA circCTNNA1 is downregulated in osteoarthritis and sponges miR-29a to suppress LPS-induced apoptosis of synoviocytes}

Wei Liu

the First Hospital of Qiqihar City

Haolong Yang

the Third Affiliated Hospital of Qiqihar

Xingyu Feng

the First Hospital of Qiqihar City

Jing Song

the First Hospital of Qiqihar City

Wei Zhong ( $\sim$ WeiZhonglmmunology@163.com )

the First Hospital of Qiqihar

\section{Research}

Keywords: osteoarthritis, circCTNNA1, miR-29a, synoviocytes, apoptosis

Posted Date: January 28th, 2021

DOI: https://doi.org/10.21203/rs.3.rs-154742/v1

License: (c) (1) This work is licensed under a Creative Commons Attribution 4.0 International License. Read Full License

Version of Record: A version of this preprint was published at Immunopharmacology and Immunotoxicology on November 9th, 2021. See the published version at https://doi.org/10.1080/08923973.2021.1988103. 


\section{Abstract}

Introduction: CircRNA circCTNNA1 has been characterized as a critical player in cancer biology, while its role in other human diseases is unknown. This study was carried out to study the role of circCTNNA1 in osteoarthritis (OA).

Materials and Methods: OA patients $(n=62)$ were subjected to extraction of synovial fluid, followed by performing RT-qPCRs to determine the expression of circCTNNA1 and miR-29a. The interaction between circCTNNA1 and miR-29a was predicted by IntaRNA 2.0 and confirmed by RNA pull-down assay. In synoviocytes, overexpression of circCTNNA1 and miR-29a was achieved, followed by performing RTqPCRs to analyze the crosstalk between them. The role of circCTNNA1 and miR-29a in regulating the apoptosis of synoviocytes was explored by cell apoptosis assay.

Results: CircCTNNA1 was downregulated in OA, while miR-29a was overexpressed in OA. CircCTNNA1 and miR-29a were not significantly correlated. RNA pull-down assay illustrated the direct interaction between circCTNNA1 and miR-29a. In synoviocytes, overexpression of circCTNNA1 and miR-29a failed to regulate the expression of each other. CircCTNNA1 overexpression suppressed the enhancing effects of miR-29a overexpression on cell apoptosis induced by LPS.

Conclusion: Therefore, circCTNNA1 is downregulates in OA and its overexpression suppresses the apoptosis of synoviocytes by sponging miR-29a.

\section{Background}

As the most common type of arthritis, osteoarthritis (OA) is caused by the chronic disruption of the protective joint cartilage that cushions bone ends $(1,2)$. OA mainly affects people after middle age. OA affects more than $13 \%$ of women and $10 \%$ of men older than 60 years $(3,4)$. OA causes chronic pain, joint stiffness and swelling $(1,2)$. At present, all available treatment approaches can only control the symptoms, such as the relief of chronic pain, while no cure is available $(5,6)$. Therefore, more effective treatment strategies are needed. However, the molecular mechanism of OA is still largely unknown.

Studies of $O A$ in recent decades have showed that $O A$ requires the participation of molecular signaling (7-10). Some molecular players, such as Notch and NF-KB pathways, have been characterized as promising molecular targets to develop targeted therapy that can be applied to treat OA by regulating gene expression (9). However, targeted therapy is still under research. Particularly, safe and effective molecular targets for OA treatment remain lacking. Circular RNAs encode no protein products, but they participate in OA by affecting protein synthesis $(11,12)$. Therefore, circRNAs may serve as potential therapeutic targets for OA. However, the role of most circRNAs in OA is unknown. CircRNA circCTNNA1 has been characterized as a critical player in cancer biology (13), while its role in other human diseases is unknown. We performed bioinformatics analysis and found that circCTNNA1 could interact with miR-29a, which is a crucial player in the apoptosis of synoviocytes (14). We then analyzed the interaction between circCTNNA1 and miR-29a in OA. 


\section{Materials And Methods}

\subsection{Research subjects and collection of synovial fluid}

First Hospital of Qiqihar City Ethics Committee approved this study. At this hospital, this study enrolled a total of $62 \mathrm{OA}$ patients (knee OA) and 62 healthy controls. Both $O A$ and control group included 22 males and 40 females, and the age was 57 to 71 years (62.8+/-5.9 years). All OA patients were excluded from recurrent cases, any initiated therapy and other clinical disorders. All OA patients and healthy controls signed informed consent. Prior to therapy, synovial fluid $(1.0 \mathrm{ml})$ extraction from affected sites of OA patients and the knee of the healthy controls was performed.

\subsection{Synoviocytes and transient transfections}

Synoviocytes (type B, primary cells) were purchased from Sigma-Aldrich (Cat. 4080A-05A). These cells are derived from an adult OA patient. Synoviocytes were cultivated in human synoviocyte media (Cell applications). Cell culture conditions were $5 \% \mathrm{CO}_{2}, 37^{\circ} \mathrm{C}$ and $95 \%$ humidity. In cases of LPS treatment, synoviocytes were treated with LPS at dosages of $0,2,4,6,8$ and $10 \mu \mathrm{g} / \mathrm{ml}$ for $48 \mathrm{~h}$ prior to the subsequent analysis.

Overexpression of circCTNNA1 and miR-29a was reached in synoviocytes by transfecting pcDNA3.1circCTNNA1 vector (Invitrogen) or miR-29a (Invitrogen) using Lipofectamine 2000 (Invitrogen). NC experiments were transfections of NC miRNA or empty pcDNA3.1 vector. Untransfected cells were used as control (C) cells. Expression vectors and miRNAs were first mixed with transfection reagent to form transfection mixtures, followed by incubation with cells for $6 \mathrm{~h}$. After that, cells were washed with fresh medium. Prior to the subsequent assays, cells were cultured for $48 \mathrm{~h}$.

\subsection{Preparation RNA samples}

RNAzol (Sigma-Aldrich) was used for the isolation of total RNAs from both synoviocytes and samples of synovial fluid from both OA and control group. DNase I (Invitrogen) was used to remove genomic DNA. After that, RNAs were separated through 5\% urea-PAGE electrophoresis to analyze RNA integrity. Ratios of OD260/280 were determined to analyze RNA purity and a ratio close to 2.0 indicated pure RNA samples.

\subsection{RT-qPCRs}

Preparation of cDNA samples was performed using 5000ng total RNA per reaction. With $18 \mathrm{~S}$ rRNA as an endogenous control, qPCRs were performed using SYBR Green Master Mix (Bio-Rad) to determine the expression of circCTNNA1.

The expression of mature miR-29a was performed by performing poly (A) addition, followed by miRNA reverse transcriptions and qPCRs with poly $(T)$ was reverse primer. All steps were completed using All-inOne ${ }^{\mathrm{TM}}$ miRNA qRT-PCR Detection Kit* (GeneCopoeia). U6 was used as the internal control of miR-29a. 
Primer sequences were: 5'-TGCGTAGACAGCTCCGCAAAG-3' (forward) and 5'-

ATCAATTTGTTGGCATGTTC-3'(reverse) for circCTNNA1; 5'-TAACCCGTTGAACCCCATT-3' (forward) and 5'CCATCCAATCGGTAGTAGC3'(reverse) for 18S rRNA. Forward primer of miR-29a was

ACTGATTTCTTTTGGTG. MiR-29a reverse primer and U6 primers were from the kit. PCR reaction conditions were: $95^{\circ} \mathrm{C}$ for $3 \mathrm{~min}$, followed by 40 cycles of $95^{\circ} \mathrm{C}$ for $10 \mathrm{~s}$ and $58^{\circ} \mathrm{C}$ for $45 \mathrm{~s}$. The method of $2^{-\Delta \Delta C t}$ was used to normalize the Ct values.

\subsection{RNA-RNA interaction prediction and RNA pull-down assay}

The interaction between circCTNNA1 and miR-29a was predicted using an online program named IntaRNA2.0. The long sequent was circCTNNA1 and the short sequent was miR-29a. Other default parameters were used.

Biotinylated NC (Bio-NC) and circCTNNA1 RNA (Bio-circCTNNA1) were synthesized by Sangon biotech (Shanghai, China). Bio-NC and Bio-circCTNNA1 were transfected into synoviocytes through the aforementioned methods. The transfected cells were transfected in fresh medium for $48 \mathrm{~h}$, followed by the preparation of cell lysis. After that, streptaviden magnetic beads (Invitrogen) were used to pull down Bio-NC and Bio-circCTNNA1, followed by RT-qPCRs to determine the expression of miR-29a.

\subsection{Western blot}

RIPA solution and BCA assays (Invitrogen) were used to isolate and quantify protein samples. Protein samples were denatured and subjected to electrophoresis using 5\% urea-PAGE gel. PVDF membranes were used to transfer proteins, followed by incubation with caspase antibody (ab2302, Abcam) and antirabbit IgG-HRP secondary antibody (1:1000, MBS435036, MyBioSource). Signals were produced with ECL Western Blotting Substrate (Thermo Fisher Scientific).

\subsection{Apoptosis assay}

Synoviocytes with transfections were transferred to a 6-well cell culture plate with $2 \mathrm{ml}$ medium containing 40,000 cells per well. Three replicate wells were set for each experiment. LPS was added to reach $10 \mu \mathrm{g} / \mathrm{ml}$ and cells were cultivated under the aforementioned methods for $48 \mathrm{~h}$. After that, AnnexinV FITC and propidium iodide (PI) staining was performed in dark for $20 \mathrm{~min}$. After that, cell apoptosis was analyzed by flowing software.

\subsection{Statistical analysis}

Comparisons between Control and OA groups were performed by unpaired t test. ANOVA (one-way or twoway) Tukey's test was used to compare multiple groups. Correlations were analyzed by Pearson's correlation coefficient analysis. $\mathrm{P}<0.05$ was statistically significant.

\section{Results}




\subsection{Altered expression of circCTNNA1 and miR-29a was observed in OA group}

Synovial fluid samples were subjected o RT-qPCRs to determine the expression of circCTNNA1 and miR29a. CircCTNNA1 was significantly downregulated in OA (Fig. 1A, $p<0.001$ ), while miR-29a was significantly overexpressed in $\mathrm{OA}$ (Fig. 1B, p < 0.001). Therefore, the overexpression of miR-29a and downregulation of circCTNNA1 may participate in OA. It is worth noting that levels of CircCTNNA1 and miR-29a expression in OA patients were close correlated with OA stages (both $p<0.05$ ), but not patients' age, gender, BMI and smoking and drinking habits.

\subsection{CircCTNNA1 and miR-29a directly interact with each other in synoviocytes}

The interaction between circCTNNA1 and miR-29a was predicted by IntaRNA2.0. It was observed that circCTNNA1 and miR-29a may form strong base pairing (Fig. 2A). RNA pull-down assay was applied to analyze the interaction between them in synoviocytes. Compared to Bio-NC group, Bio-circCTNNA1 group exhibited significantly higher level of miR-29a expression (Fig. 2B, $p<0.05$ ). Therefore, circCTNNA1 and miR-29a can interact with each other in synoviocytes.

\subsection{CircCTNNA1 and miR-29a failed to regulate the expression of each other in synoviocytes}

CircCTNNA1 and miR-29a were significantly overexpressed in synoviocytes between $24 \mathrm{~h}$ and $96 \mathrm{~h}$. Interestingly, overexpression of circCTNNA1 and miR-29a failed to regulate the expression of each other (Fig. 3B). Moreover, ircCTNNA1 and miR-29a were not significantly correlated across OA (Fig. 3C) and control (Fig. 3D) samples.

\subsection{CircCTNNA1 may sponge miR-29a to reduce the apoptosis of synoviocytes induced by LPS.}

Synoviocytes were treated with LPS at dosages of $0,2,4,6,8$ and $10 \mu \mathrm{g} / \mathrm{ml}$ for $48 \mathrm{~h}$. The experiments illustrated that LPS significantly downregulated circCTNNA1 (Fig. 4A, $p<0.05$ ) and significantly upregulated miR-29a (Fig. 4B, p < 0.05) in synoviocytes. The role of circCTNNA1 and miR-29a in regulating the apoptosis of synoviocytes induced by LPS $(10 \mu \mathrm{g} / \mathrm{ml}$ for $48 \mathrm{~h})$ was analyzed by cell apoptosis. Moreover, circCTNNA1 decreased and miR-29a increased cell apoptosis. In addition, circCTNNA1 suppressed the role of miR-29a in enhancing cell apoptosis induced by LPS (Fig. 4C, $p<$ 0.05). Expression of active (cleaved) caspase-3 in different cell apoptosis groups were analyzed by Western blot. Levels of active caspase-3 were in accordance with the levels of cell apoptosis (Fig. 4D).

\section{Discussion}


The crosstalk between circCTNNA1 and miR-29a was explored in OA. We found that circCTNNA1 was downregulated in OA. In addition, circCTNNA1 may sponge miR-29a to reduce the enhancing effects of miR-29a on the apoptosis of synoviocytes induced by LPS.

CircCTNNA1 has been characterized as a critical player in colorectal cancer (13). It is reported that circCTNNA1 is overexpressed in colorectal cancer, and it upregulate FOXM1 by sponging miR-149-5p to promote cancer progression (13). However, the role of circCTNNA1 in other human disease is unknown. In this study we observed the downregulation of circCTNNA1 in OA, suggesting the involvement of circCTNNA1 in this disease. Type B synoviocytes are responsible for the production of glycoproteins in the synovial fluid, which is essential for joint lubrication. However, under disease conditions, the activation of type B synoviocytes by proinflammatory cytokines may increase the production of erosive enzymes, such as collagenase, GM-CSF, aggrecanase and metalloproteinases in these cells, to increase joint destruction and cartilage degradation, which in turn aggregate the conditions of $O A(15,16)$. In this study we showed that circCTNNA1 reduced the apoptosis of type B synoviocytes induced by LPS. Therefore, circCTNNA1 may participate in OA by regulating cell apoptosis.

MiR-29a plays critical roles in rheumatoid arthritis (RA) by inducing the apoptosis of synoviocytes and inhibiting cell proliferation (14). Interestingly, our study also observed the overexpression of miR-29a in $\mathrm{OA}$ and its enhancing effects on the apoptosis of OA-derived synoviocytes(17). Therefore, miR-29a may participate in both OA and RA, which have opposite pathological changes.

Interestingly, our study showed that circCTNNA1 and miR-29a could directly interact with each other in synoviocytes. However, overexpression of circCTNNA1 and miR-29a failed to significantly affect the expression of each other. Instead, circCTNNA1 overexpression reduced the enhancing effects of miR-29a on cell apoptosis. Therefore, we speculate that circCTNNA1 may sponge miR-29a to suppress its role in $\mathrm{OA}$.

\section{Conclusion}

In conclusion, circCTNNA1 is under-expressed in OA and it may sponge miR-29a to reduce the apoptosis of type B synoviocytes induced by LPS.

\section{Declarations}

\subsection{Ethical Approval and Consent to participate}

Informed consent was obtained from all individual participants included in the study. All producers were approved by the First Hospital of Qiqihar City Ethics Committee. Procedures operated in this research were completed in keeping with the standards set out in the Announcement of Helsinki and laboratory guidelines of research in China.

\subsection{Code availability}


Not Applicable.

\subsection{Consent to publish}

Not Applicable.

\subsection{Availability of supporting data}

All data was presented in this manuscript.

\subsection{Competing interests}

No competing interest to declare.

\subsection{Funding}

This work was supported by the Qiqihar Science and Technology Planning Project (Grant No. SFGG201924) and Natural Science Foundation of Heilongjiang Province (No. LH2020H135)

\subsection{Authors' contributions}

Wei Liu, Haolong Yang and Wei Zhong: designed and performed experiments, manuscript preparation; Xingyu Feng and Jing Song: performed data analysis.

All authors have read and approve the submission of the manuscript.

\subsection{Acknowledgements}

We thank the Qiqihar Science and Technology Planning Project and Natural Science Foundation of Heilongjiang Province for approving.

\section{References}

1. Glyn-Jones S, Palmer AJ, Agricola R, Price AJ, Vincent TL, Weinans H, et al. Osteoarthritis. Lancet. 2015;386(9991):376-87.

2. Vina ER, Kwoh CK. Epidemiology of osteoarthritis: literature update. Current opinion in rheumatology. 2018;30(2):160-7.

3. Rahmati M, Nalesso G, Mobasheri A, Mozafari M. Aging and osteoarthritis: Central role of the extracellular matrix. Ageing research reviews. 2017;40:20-30.

4. Loeser RF. The Role of Aging in the Development of Osteoarthritis. Transactions of the American Clinical and Climatological Association. 2017;128:44-54.

5. Özkuk K, Uysal B. Is the Duration of Spa Cure Treatment Important in Knee Osteoarthritis? A Randomized Controlled Study. Complementary medicine research. 2019;26(4):258-64. 
6. Anandacoomarasamy A, March L. Current evidence for osteoarthritis treatments. Therapeutic advances in musculoskeletal disease. 2010;2(1):17-28.

7. Lee AS, Ellman MB, Yan D, Kroin JS, Cole BJ, van Wijnen AJ, et al. A current review of molecular mechanisms regarding osteoarthritis and pain. Gene. 2013;527(2):440-7.

8. de Campos GC, Tieppo AM, de Almeida CS, Jr., Hamdan PC, Alves WM, de Rezende MU. Target-based approach for osteoarthritis treatment. World journal of orthopedics. 2020;11(6):278-84.

9. Saito T, Tanaka S. Molecular mechanisms underlying osteoarthritis development: Notch and NF-KB. Arthritis research \& therapy. 2017;19(1):94.

10. Mobasheri A. The future of osteoarthritis therapeutics: targeted pharmacological therapy. Current rheumatology reports. 2013;15(10):364.

11. Yu CX, Sun S. An Emerging Role for Circular RNAs in Osteoarthritis. Yonsei medical journal. 2018;59(3):349-55.

12. Wang Y, Wu C, Zhang F, Zhang Y, Ren Z, Lammi MJ, et al. Screening for Differentially Expressed Circular RNAs in the Cartilage of Osteoarthritis Patients for Their Diagnostic Value. Genetic testing and molecular biomarkers. 2019;23(10):706-16.

13. Chen P, Yao Y, Yang N, Gong L, Kong Y, Wu A. Circular RNA circCTNNA1 promotes colorectal cancer progression by sponging miR-149-5p and regulating FOXM1 expression. Cell death \& disease. 2020;11(7):557.

14. Liu J, Fei D, Xing J, Du J. MicroRNA-29a inhibits proliferation and induces apoptosis in rheumatoid arthritis fibroblast-like synoviocytes by repressing STAT3. Biomedicine \& pharmacotherapy = Biomedecine \& pharmacotherapie. 2017;96:173-81.

15. Castrogiovanni P, Di Rosa M, Ravalli S, Castorina A, Guglielmino C, Imbesi R, et al. Moderate Physical Activity as a Prevention Method for Knee Osteoarthritis and the Role of Synoviocytes as Biological Key. International journal of molecular sciences. 2019;20(3).

16. Hirohata S, Nagai T, Asako K, Tomita T, Yoshikawa H. Induction of type B synoviocyte-like cells from plasmacytoid dendritic cells of the bone marrow in rheumatoid arthritis and osteoarthritis. Clinical immunology (Orlando, Fla). 2011;140(3):276-83.

17. Withrow J, Murphy C, Liu Y, Hunter M, Fulzele S, Hamrick MW. Extracellular vesicles in the pathogenesis of rheumatoid arthritis and osteoarthritis. Arthritis research \& therapy. 2016;18(1):286.

\section{Figures}



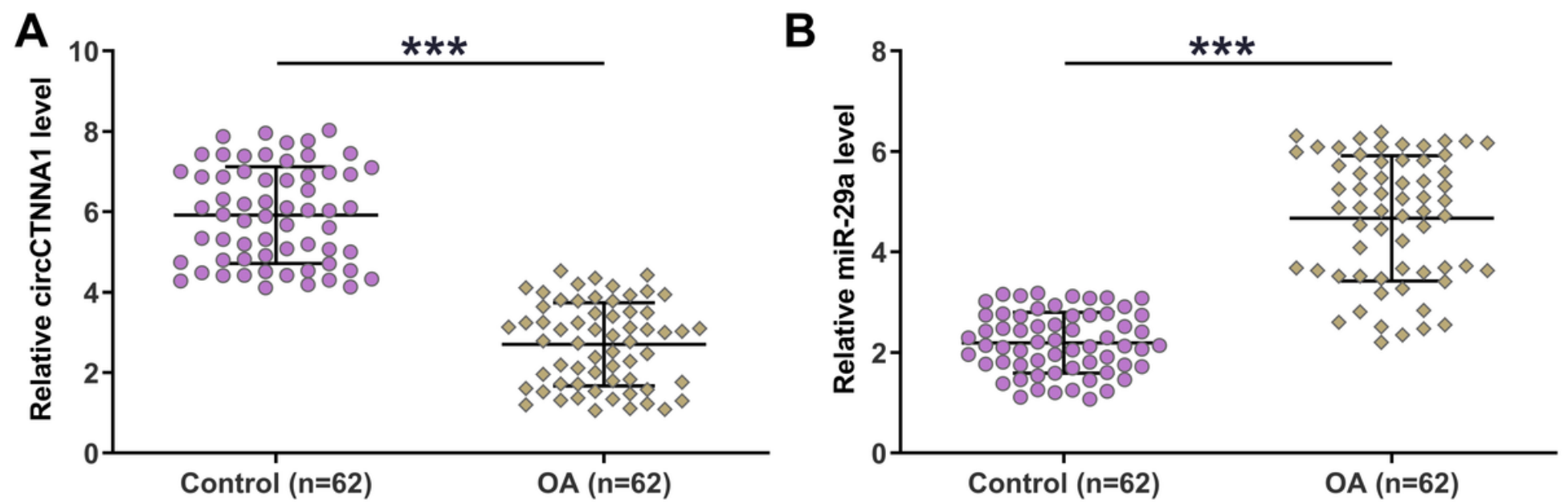

Figure 1

Altered expression of circCTNNA1 and miR-29a was observed in OA group The 62 OA patients and 62 healthy controls were subjected to extraction of synovial fluid, followed by performing RT-qPCRs to determine the expression of circCTNNA1 and miR-29a. The average values of three qPCR replicates were present.***, $p<0.001$
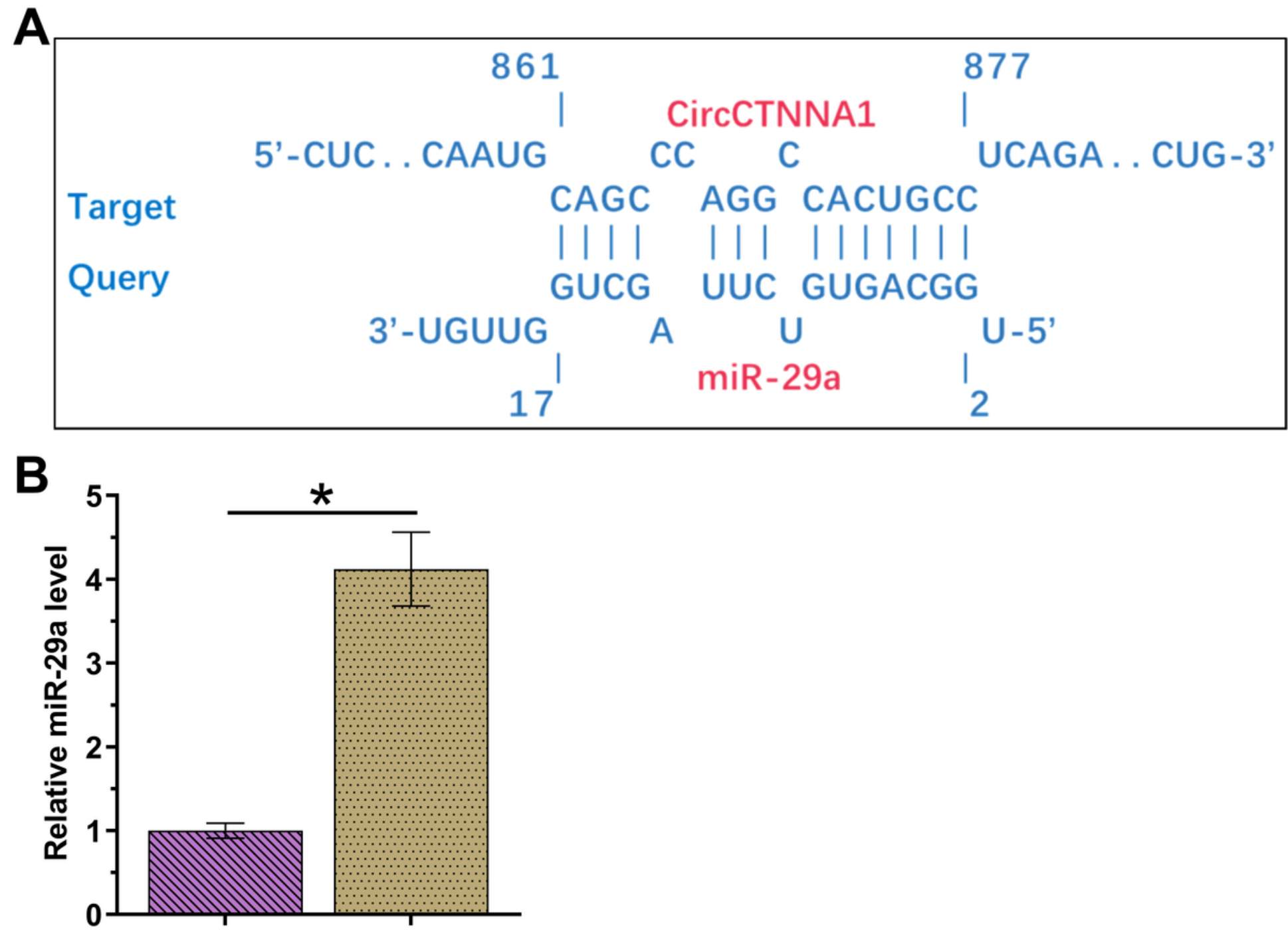

Bio-NC Bio-circCTNNA1 
Figure 2

CircCTNNA1 and miR-29a directly interact with each other in synoviocytes The interaction between circCTNNA1 and miR-29a was predicted by IntaRNA2.0 (A). RNA pull-down assay was applied to analyze the interaction between them in synoviocytes $(B) .{ }^{*}, p<0.05$.
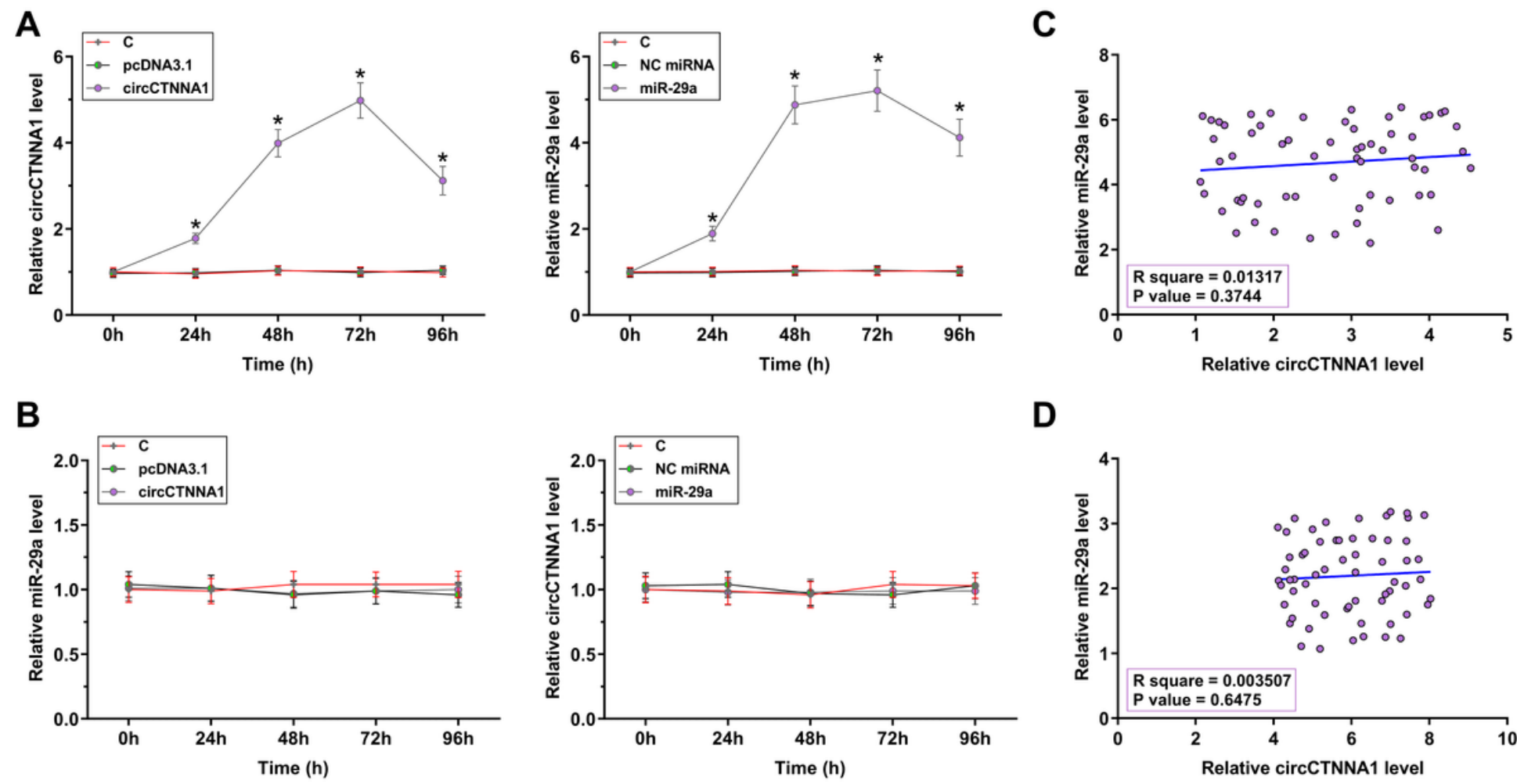

D

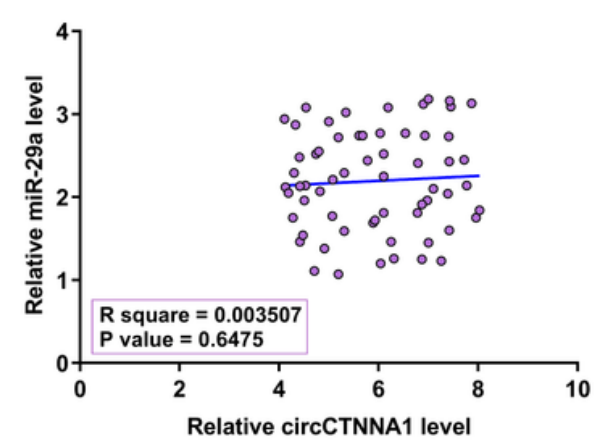

Figure 3

CircCTNNA1 and miR-29a failed to regulate the expression of each other in synoviocytes To explore the interaction between circCTNNA1 and miR-29a, synoviocytes were transfected with either circCTNNA1 expression vector or mimic of miR-29a, followed by the confirmation of overexpression every $24 \mathrm{~h}$ until 96h (B). The effects of circCTNNA1 and miR-29a overexpression on the expression of each other were analyzed by RT-qPCR (B). The correlations between circCTNNA1 and miR-29a were analyzed by Pearson's correlation coefficient across both $\mathrm{OA}(\mathrm{C})$ and control (D) synovial fluid samples. ${ }^{*}, \mathrm{p}<0.05$. 


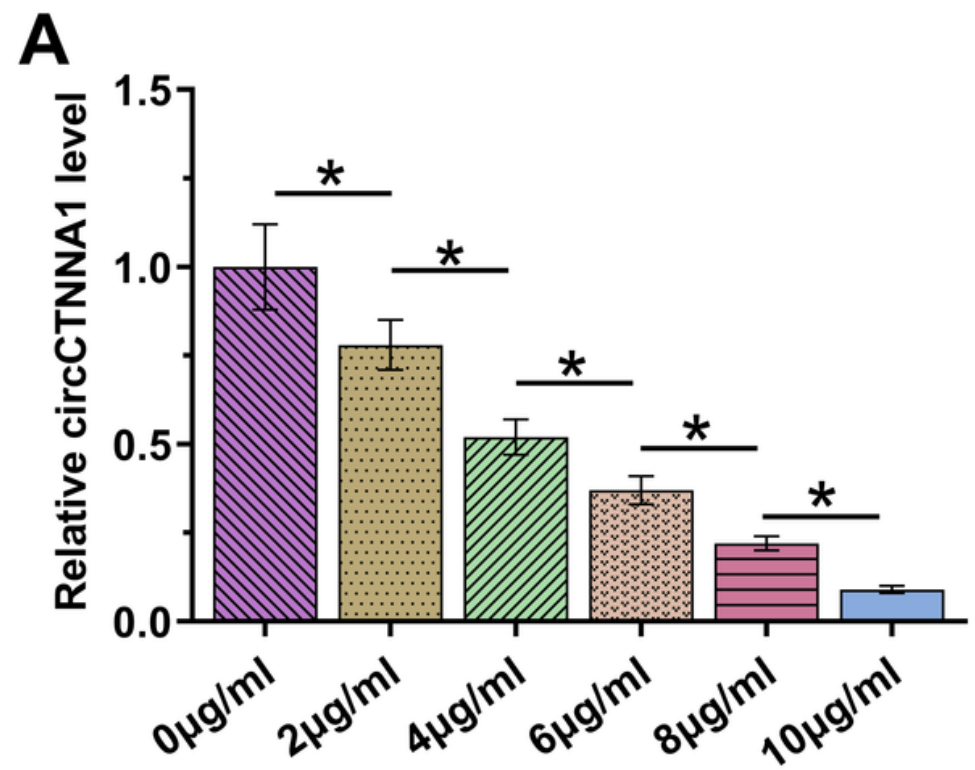

B

C
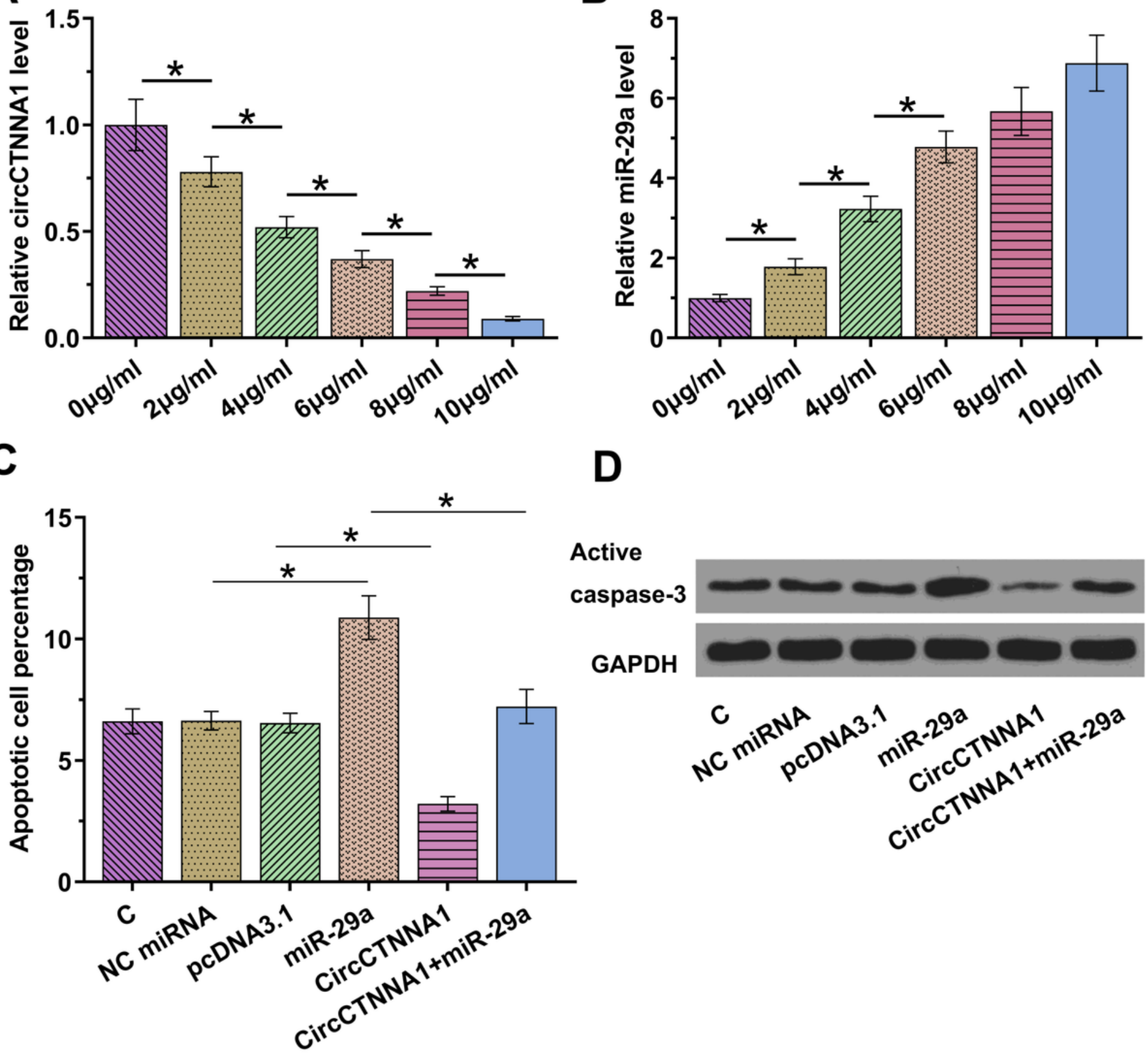

D

Active

caspase-3

GAPDH
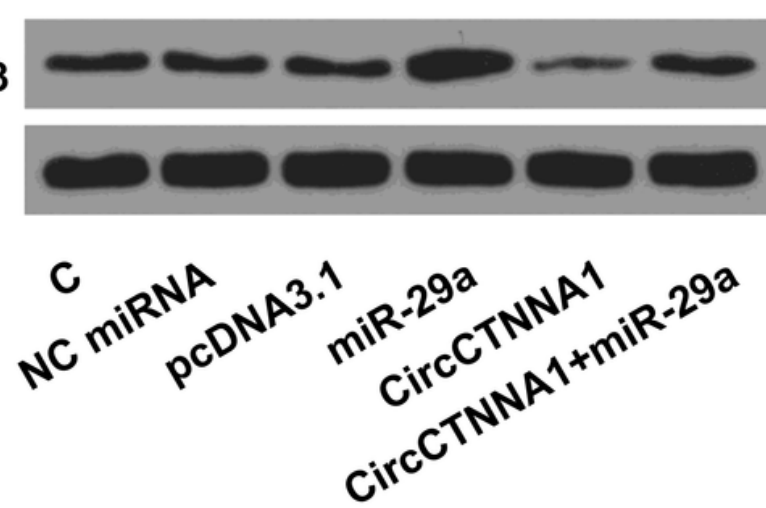

Figure 4

CircCTNNA1 may sponge miR-29a to reduce the apoptosis of synoviocytes induced by LPS. Synoviocytes were treated with LPS at dosages of $0,2,4,6,8$ and $10 \mu \mathrm{g} / \mathrm{ml}$ for $48 \mathrm{~h}$, followed by the determination of the expression of circCTNNA1 (A) and miR-29a (B) by RT-qPCRs (A). The role of circCTNNA1 and miR-29a in regulating the apoptosis of synoviocytes induced by LPS $(10 \mu \mathrm{g} / \mathrm{ml}$ for $48 \mathrm{~h})$ was analyzed by cell apoptosis (C). Expression of active (cleaved) caspase-3 in different cell apoptosis groups were analyzed by Western blot $(D) .{ }^{*}, p<0.05$. 